\title{
Perfil emprendedor de los estudiantes de la carrera de Economía de la Universidad Técnica de Machala en presencia del COVID-19
}

\section{Entrepreneurial profile of the students of the Economics career of the Technical University of Machala in the presence of COVID-19}

John Alexander Campuzano-Vásquez

Universidad Técnica de Machala, Machala, Ecuador

jcampuzano@utmachala.edu.ec

iD http://orcid.org/0000-0002-3901-3197

Luis Fernando Cedillo-Chalaco

Investigador Independiente, Machala, Ecuador

lfcc_lye@hotmail.com

https://orcid.org/0000-0002-3142-4485

Recepción: 20/09/2021 | Aceptación: 09/1 1/2021 | Publicación: 25/11/2021

Cómo citar (APA, séptima edición):

Campuzano-Vásquez, J.A., y Cedillo-Chalaco, L.F. (2021). Perfil emprendedor de los estudiantes de la carrera de Economía de la Universidad Técnica de Machala en presencia del

COVID-19. INNOVA Research Journal, 6(3.2), 47-62.

https://doi.org/10.33890/innova.v6.n3.2.2021.1939

\section{Resumen}

Las universidades y los institutos tecnológicos ecuatorianos vienen desde hace algún tiempo implementando estrategias educativas curriculares para fomentar el emprendimiento entre sus educandos, bien sea, mediante asignaturas, concursos, ferias, etc., el énfasis de estas propuestas sigue direccionándose a las carreras empresariales e industriales. La presencia del virus pandémico COVID-19 desde el año 2020, ha provocado un retroceso en los mercados mundiales y locales, aumento del desempleo, reducción de ventas y desaceleración de la dinámica empresarial ecuatoriana; por lo que se hace necesario identificar si la presencia de esta enfermedad ha incidido en el perfil emprendedor de quienes acuden a las universidades a formarse; para ello, se utilizó una metodología de enfoque cuantitativo, con alcance descriptivo y correlacional, que permitió evaluar el perfil emprendedor de los estudiantes de la Carrera de Economía de la Universidad Técnica de Machala. Para alcanzar este objetivo se usó un test de actitudes que se basa en 29 preguntas y otras tantas sub preguntas orientadas a la creación de empresas. Este test fue usado en España y Puerto 
Rico en varias universidades por Veciana y Urbano en 2004 y que ha sido adaptado para la presente investigación. Se encuentra que, los estudiantes se perciben como emprendedores, poseen un adecuado perfil emprendedor (aunque ambas correlacionen moderadamente), y la no existencia de diferencia estadísticamente significativa en el perfil emprendedor entre los estudiantes de todos los semestres analizados.

Palabras claves: emprendedores; emprendimiento; economía de la ciencia.

\begin{abstract}
Ecuadorian universities and technological institutes have been implementing curricular educational strategies for some time to promote entrepreneurship among their students, either through courses, competitions, fairs, etc., the emphasis of these proposals continues to be directed to business and industrial careers. The presence of the COVID-19 pandemic virus since 2020 has caused a setback in world and local markets, increased unemployment, reduced sales and a slowdown in Ecuadorian business dynamics; Therefore, it is necessary to identify whether the presence of this disease has had an impact on the entrepreneurial profile of those who go to universities for training; For this, a quantitative approach methodology was used, with a descriptive and correlational scope, which allowed evaluating the entrepreneurial profile of the students of the Economics Career of the Technical University of Machala. To achieve this objective, an attitudes test was used, which is based on 29 questions and as many sub-questions aimed at business creation. This test was used in Spain and Puerto Rico in several universities by Veciana and Urbano in 2004 and it has been adapted for the present investigation. It is found that the students are perceived as entrepreneurs, have an adequate entrepreneurial profile (although both correlate moderately), and the non-existence of a statistically significant difference in the entrepreneurial profile among the students of all the semesters analyzed.

Keywords: entrepreneurs; entrepreneurship; economics of science.
\end{abstract}

\title{
Introducción
}

Los países que trabajan por mejorar las condiciones de vida de sus habitantes ven en el emprendimiento una oportunidad para ello. Los empleos e ingresos generados por la apertura de nuevas empresas hacen del emprendimiento un fenómeno socioeconómico destacado a todo nivel y vigente en el tiempo, (y más ahora con la presencia de una pandemia denominada COVID-19) que despierta el interés de gobiernos e instituciones privadas por los evidentes resultados. Los emprendedores no son personas diferentes a otras, o que tengan atributos fuera de lo común, sino que actúan como personas dinámicas que buscan nuevas combinaciones o innovaciones acerca de lo que ven y usan habitualmente, y que con sus acciones causan inestabilidad en el mercado (Schumpeter, 1942).

Además, el fenómeno emprendimiento puede definirse, dentro de las múltiples acepciones que existen del mismo, como el desarrollo de un proyecto que persigue un determinado fin económico, político o social, entre otros, y que posee ciertas características, principalmente que tiene una cuota de incertidumbre y de innovación (Formichella, 2004). Esas características llevan a configurar un espíritu emprendedor estudiado por Veciana et al. (2005) que identifica las percepciones de los estudiantes universitarios sobre la conveniencia y la viabilidad de crear nuevas empresas como primer paso para despertar y estimular el interés de los estudiantes por la carrera empresarial. 
Desde lo educativo, los centros de enseñanza superior tratan de determinar los aspectos que influyen en los comportamientos emprendedores para crear programas de preferencia en especialidades empresariales que unen una serie de actividades enfocadas a lo práctico (Bagheri y Lope Pihie, 2013). Desde sus bases conceptuales, la cooperación entre la universidad y los actores públicos, y privados, implica compromiso social, y comunitario, y el desarrollo de actividades innovadoras (Vera Salazar et al., 2013), compromiso que para Harrison y Turok (2017) genera una mayor participación de académicos con la comunidad empresarial externa, la sociedad civil y diferentes partes del gobierno. Estas relaciones se ven reflejadas en las funciones sustantivas de las universidades ecuatorianas artículo 117 de Ley Orgánica de Educación Superior y descritas como: enseñanza, investigación y la vinculación con la sociedad (CEAACES, 2018).

Para (Febriyantoro, 2019; Malinda, 2019) es un reto para las universidades producir graduados que puedan contribuir a la creación del campo de trabajo y para ello, es necesario el crecimiento del espíritu emprendedor en los estudiantes a través de la educación empresarial.

Al tener estas delicadas funciones las universidades, tienen que promover entre sus educandos algunos comportamientos que se identifican con un emprendedor, como es el ser un individuo líder, previsor, tomador de riesgos, que auto aprende, que es evaluador de oportunidades, y que moviliza recursos desde una zona de bajo rendimiento a una de alta productividad (Burnett, 2000), comportamientos que no han cambiado mucho en las dos últimas décadas en el mundo y en Ecuador, a estos comportamientos se pueden adicionar, el manejo eficaz de redes sociales, de técnicas de marketing digital y de tecnología aplicada.

En Ecuador, algunos autores como (Morán y Sánchez, 2018; Víctor et al., 2019) vienen trabajando en universidades privadas y públicas para determinar el perfil emprendedor y los elementos, que permiten desarrollar emprendimientos como salida profesional, se tienen resultados comunes, como incipiente actividad emprendedora y estrategias débiles de apoyo desde la percepción estudiantil con porcentajes bajos de asistencia a cursos o capacitaciones. Hay que resaltar que estos estudios se desarrollan entre estudiantes de diferentes edades y años de estudios. Los sectores de emprendimiento más relevantes están en lo comercial, y se indica que se necesita una mayor cohesión entre los conocimientos recibidos y la actividad real.

Para tener una aproximación a la realidad estudiantil universitaria dentro del Ecuador, es importante referirse al informe GEM 2019-2020, el cual indica que el perfil de los emprendedores ecuatorianos, mayoritariamente está en los hombres con el 52.2\%, la edad promedio para emprender es de 36.23 años, que resulta muy elevada a la edad promedio de los estudiantes universitarios, lo que puede llevar a pensar que hay una inclinación a buscar oportunidades luego de salir de los centros de educación; la escolaridad promedio es de 12.28 años (secundaria terminada), y el ingreso del hogar está por debajo de los 2 salarios unificados básicos -esto para los que son parte de la TEA ${ }^{1}$ - (Lasio et al., 2020).

\footnotetext{
${ }^{1}$ Actividad Emprendedora Temprana (TEA), que incluye a todas las personas adultas que están en el proceso de iniciar un negocio o han iniciado uno que no ha sobrepasado del umbral de 42 meses. Específicamente, la TEA se compone de dos tipos de emprendedores: emprendedores nacientes y nuevos.
} 
Las motivaciones para emprender están presentes en la necesidad de acumular riqueza, hacer la diferencia, escasez de empleo y tradición familiar en función de la tasa de emprendimiento temprano, objetivos que no se pueden determinar con certeza en los estudiantes universitarios que en tiempos de pandemia pueden inclinarse a la creación de su propio empleo.

Se puede observar que no hay una conexión fuerte entre el emprendimiento a nivel superior con las estadísticas del reporte GEM, es por ello, que la investigación que realiza Honig (2004) muestra desacuerdos teóricos y empíricos al momento de analizar la educación emprendedora y las intenciones emprendedoras. Que es lo que se plantea en las universidades y que ha llevado a diseñar reformas dentro de las mallas curriculares, creando asignaturas directamente relacionadas con la capacidad de emprender. ¿Existe o no relación entre la educación que reciben los estudiantes universitarios y su intención de emprender? La duda, se reduce con la presencia de una pequeña correlación positiva entre la educación y la intención emprendedora (Martin et al., 2013).

De este modo, el número de emprendimientos o de propuestas realizadas por los estudiantes universitarios, no solo se deben considerar para determinar la eficiencia de la educación emprendedora, hay que tratar de revisar si las actividades educativas corresponden al perfil del emprendedor que acude a las aulas, pero ante ello, surge una nueva pregunta: ¿existen perfiles actualizados de emprendimiento de estudiantes que acuden a las universidades? $\mathrm{O}$ solo, se cree que algunas actividades bien diseñadas son suficientes para promover el emprendimiento.

No obstante, Haase y Lautenschläger (2011) no están de acuerdo con la eficacia de la educación empresarial para aumentar la conciencia empresarial y aumentar el número de empresas. Sugieren que los programas deben enfatizar las habilidades sociales y no cómo iniciar un negocio.

Se puede entonces enseñar a tener un espíritu empresarial, Charrón y Rivera (2020) revisando trabajos de varios investigadores, indican que se puede desde la enseñanza del emprendimiento promover actitudes como la autonomía, la creatividad, la innovación o la asunción de riesgos y el acto de aventurarse a crear. Aunque, hay críticas en cuanto a la coherencia entre los objetivos y métodos de enseñanza del emprendimiento.

Para López et al. (2021) analizando varios estudios, indican que existen pocas pruebas sobre los efectos que un programa sobre emprendimiento tiene sobre las personas, y qué cosas del programa pueden ser determinantes a la hora de influir en las propuestas emprendedoras; incluso se manifiesta que los estudiantes ya llevan en su mente la predisposición de emprender, desean ser emprendedores, y buscan programas, ayudas, y toda información que les permita alcanzar su objetivo.

Bernal y Cárdenas (2017) mencionan la presencia de factores personales relacionados con la dimensión emprendedora, por lo que los programas de emprendimiento o las asignaturas que se crean para el efecto en las universidades públicas y privadas deben revisar las variables individuales, que se pueden encontrar en los levantamientos de perfiles. Con lo que debemos replantear, si estas asignaturas deben tener una nota aprobatoria o deben ser de divulgación y no calificadas, ya que no se puede obligar a los estudiantes a ser emprendedores si no tienen iniciativa, actitud y capacidad emprendedora, u otras características mínimas que se han mencionado líneas atrás. 
Para contribuir a este campo de investigación, se considera que es importante estudiar cuáles son las variables individuales asociadas con un marcado perfil emprendedor en la Carrera de Economía de la Universidad Técnica de Machala (UTMACH), que a sabiendas de que no existe un estudio similar en otras carreras, lo convierte en pionero en esta línea, dotándolo de importancia para plantear como objetivo analizar el perfil emprendedor de los estudiantes de la carrera de economía de la Universidad Técnica de Machala, a través de la aplicación de estadísticos descriptivos e inferenciales (prueba de Pearson y test de Kruskal Wallis) a los principales componentes del cuestionario sobre actitudes hacia la creación de empresas de Veciana y Urbano (2018), que ha sido aplicado con mucho éxito a más de 33.000 estudiantes en universidades de España y Puerto Rico.

\section{Metodología}

Al ser una investigación que analizó, describió y relacionó las variables que son parte del perfil emprendedor de los estudiantes de la Carrera de Economía de la UTMACH, se planteó un estudio de enfoque cuantitativo (Sánchez, 2019), con alcance descriptivo y correlacional (Niño, 2019), de corte transversal (Rodríguez y Mendivelso, 2018), y diseño de tipo no experimental (Hernández, Fernández, y Baptista, 2018).

Para lo cual, y con el objetivo de analizar el perfil emprendedor de los estudiantes en mención, se utilizó los principales ítems del cuestionario de actitudes hacia la creación de empresas de (Veciana y Urbano, 2004), aplicado en la III edición de una investigación realizada en la Universidad de Córdova por (Rodriguez y Fuentes, 2013).

Los ítems utilizados para el fin de esta investigación (ver Anexo), fueron dos de estructura cerrada, que se responde con sí o no; una de escala, que se responde marcando del 1 (muy poco emprendedora) al 10 (muy emprendedora), y veinte de tipo Likert de cuatro puntos (no, nunca; bastante, a menudo; poco, alguna vez; sí, siempre) que engloban el perfil emprendedor, las cuales presentaron un alfa de Cronbach (Tabla 1) de 0,95, que según lo expresado por Chaves y Rodríguez (2018), significa que tienen un excelente nivel de consistencia interna.

\section{Tabla 1}

Coeficiente de alfa de Cronbach

\begin{tabular}{cc}
\hline \multicolumn{2}{c}{ Alfa de Cronbach } \\
\hline Nivel de & $\mathrm{N}$ \\
fiabilidad & \\
0,95 & 20 \\
\hline
\end{tabular}

En lo que respecta a la muestra, se la determinó usando la técnica de muestreo aleatoria simple (Herrera y Konic, 2017; Otzen y Manterola, 2017), del total poblacional $(n=375)$ de estudiantes que cursan la Carrera de Economía, aplicando la fórmula de estimación muestral proporcional cuando la población es finita: 


$$
n=\frac{Z^{2} N p q}{p q Z^{2}+(N-1) e^{2}}
$$

De la cual, para efectos de mayor ajuste se usaron los valores sugeridos por Valdivieso, Valdivieso, y Valdivieso (2011): $\mathrm{p}=$ probabilidad de éxito, 50\%; q= probabilidad de fracaso, 50\%; $\mathrm{N}=$ tamaño de la población, 375 estudiantes; e= error de muestreo, 5\%; y, Z= nivel de confianza $(\alpha / 2)$ al 5\%=1,96; parámetros que, para García, Reding, y López (2013), aseguran el rigor, la relevancia, y el poder estadístico del muestreo.

Por lo tanto, al reemplazar dichos valores en los parámetros resulta un tamaño muestral de:

$$
n=\frac{(1,96)^{2}(375)(0,50)(0,50)}{(0,50)(0,50)(1,96)^{2}+(375-1)(0,05)^{2}}=190 \text { estudiantes }
$$

Por último, para facilitar el análisis estadístico, y la descripción e inferencia de las variables que conforman la data, se aplicó en lo que respecta a la estadística descriptiva, medidas de frecuencias absoluta y relativa, y medidas de tendencia central (León y Pérez, 2019); en cambio, para el análisis estadístico inferencial, se usó para medir el nivel de la asociación entre el perfil emprendedor y la autovaloración emprendedora, la prueba de correlación de Pearson (Hernández, et al., 2018), considerado uno de los test de mayor empleo para la evaluación de la asociación entre variables (Simancas y Arévalo, 2017); y para medir diferencias estadísticas del perfil emprendedor en cada semestre de la carrera de economía, la prueba no paramétrica para el análisis de varianza Kruskal Wallis (Flores, et al., 2017), la cual goza de amplia aplicabilidad en diferentes ciencias, debido a su versatilidad, y porque utiliza un mínimo de supuestos estadísticos (Núñez, 2018). Los datos fueron procesados mediante el software estadístico SPSS versión 24.

\section{Resultados}

De acuerdo a los datos analizados, se obtuvo respecto a la información sociodemográfica (Tabla 2), que de la muestra tomada conformada por los estudiantes de la Carrera de Economía, la mayor parte de estos, con una frecuencia absoluta de $115(60,53 \%)$ son de sexo femenino, mientras que $75(39,47 \%)$ son de sexo masculino; de los cuales, $132(69,47 \%)$ estudiantes tienen edades que oscilan entre los 20 y 25 años; seguido de un grupo de 43 (22,63\%) alumnos, que están por debajo de los 20 años; el restante de estudiantes, $14(7,37 \%)$ y $1(0,53 \%)$ poseen edades respectivas, entre 26 y 35 años, y más de 35 años.

Además, la Tabla 2 muestra información del número de estudiantes que pertenecen a los diferentes semestres de la Carrera de Economía, en donde, los niveles que más estudiantes tienen son: séptimo, sexto, primero, y tercero, con valores respectivos de 33 (17,36\%), 29 (15,26\%), 28 $(14,74 \%)$, y $25(13,15 \%)$; seguido por los semestres: quinto, segundo, y octavo con frecuencias distribuidas respectivamente de $22(11,58 \%), 21(11,05 \%)$, y $21(11,05 \%)$; y finalmente, el cuarto semestre con el menor número de sujetos $11(5,79 \%)$. 
Perfil emprendedor de los estudiantes de la carrera de Economía de la Universidad Técnica de Machala en presencia del COVID-19

Tabla 2

Información sociodemográfica de la muestra

\begin{tabular}{|c|c|c|c|c|c|c|c|}
\hline & Variable & $\mathbf{n}$ & $\%$ & & Variable & $\mathrm{n}$ & $\%$ \\
\hline \multirow[t]{4}{*}{ Sexo } & & & & Semestre & & & \\
\hline & Masculino & 75 & 39,47 & & Primer semestre & 28 & 14,74 \\
\hline & Femenino & 115 & 60,53 & & Segundo semestre & 21 & 11,05 \\
\hline & & & & & Tercer semestre & 25 & 13,16 \\
\hline \multirow[t]{5}{*}{ Edad } & & & & & Cuarto semestre & 11 & 5,79 \\
\hline & Menos de 20 años & 43 & 22,63 & & Quinto semestre & 22 & 11,58 \\
\hline & Entre 20 y 25 años & 132 & 69,47 & & Sexto semestre & 29 & 15,26 \\
\hline & Entre 26 y 35 años & 14 & 7,37 & & Séptimo semestre & 33 & 17,36 \\
\hline & Más de 35 años & 1 & 0,53 & & Octavo semestre & 21 & 11,05 \\
\hline
\end{tabular}

Por otra parte, y siguiendo con lo datado en el objetivo investigativo, en la Tabla 3, se observan los valores de centralización del nivel de autoevaluación emprendedora, en la que, según la naturaleza de la pregunta, se valora del 1 (muy poco emprendedora) al 10 (muy emprendedora); en donde los estudiantes de economía, en promedio, registraron un valor de 7,68 puntos, lo que se traduce a que se consideran personas bastante emprendedoras, desde el sentido de tener la destreza y capacidad de poner en función ideas e iniciativas para la gesta de emprendimientos; lo cual es apoyado por (González, et al., 2017), quienes expresan que las competencias para que una persona se convierta en emprendedora, depende sustancialmente de la autovaloración que el individuo tiene hacia sí mismo. Adicionalmente, la puntuación entre cada alumno, en relación con la media, presentó una desviación estándar de 1,57 puntos; pese a que se registró por parte de uno de ellos una puntuación de 2 (outlier). Este comportamiento no se muestra ajeno a lo expuesto por (Rodriguez y Fuentes, 2013).

\section{Tabla 3}

Medidas de tendencia central del nivel de autoevaluación emprendedora

\begin{tabular}{lr}
\hline Parámetro & \multicolumn{1}{c}{ Valor } \\
\hline Media & 7,68 \\
Mediana & 8,00 \\
Moda & 8 \\
Desviación & 1,573 \\
estándar & 2 \\
Mínimo & 10 \\
Máximo & \\
\hline
\end{tabular}

En complemento, la Figura 1, expresa a por menor la frecuencia de las puntuaciones marcadas por los estudiantes, en lo que refiere a la autoevaluación emprendedora; en donde la 
mayor parte de los alumnos de economía ( $n=158$ ), que significan el 83,16\% de la muestra, se autoevaluaron con una puntuación por encima de 7/10. De forma particular, de los 158 estudiantes y con la frecuencia más alta, 53 sujetos se autocalificaron con 8 puntos; seguido de 47 chicos que precisaron una puntuación de 7; y finalmente, 35 y 23 aprendices que se calificaron con 9 y 10 puntos, respectivamente. En detrimento, 32 alumnos, que representan el 16,84\% del total muestral, manifestaron una autoevaluación emprendedora, por debajo de los 6 puntos. Es preciso destacar, que la óptima autovaloración que presentaron los sujetos foco de estudio se debe, a que durante su etapa universitaria obtienen aptitudes y competencias empresariales (González, et al., 2018), a través de la formación en temáticas como: contabilidad, negocios, emprendimiento, finanzas, costos, tributación, entre otros (Boza, et al., 2020); junto con la conducta, el control percibido, y la norma subjetiva de la intención emprendedora (Bravo, et al., 2021).

\section{Figura 1}

\section{Puntuación de autoevaluación emprendedora}

En una escala de 1 a 10, determina en qué medida te sientes una persona emprendedora, llena de ideas e iniciativas para poner en práctica en su trabajo.

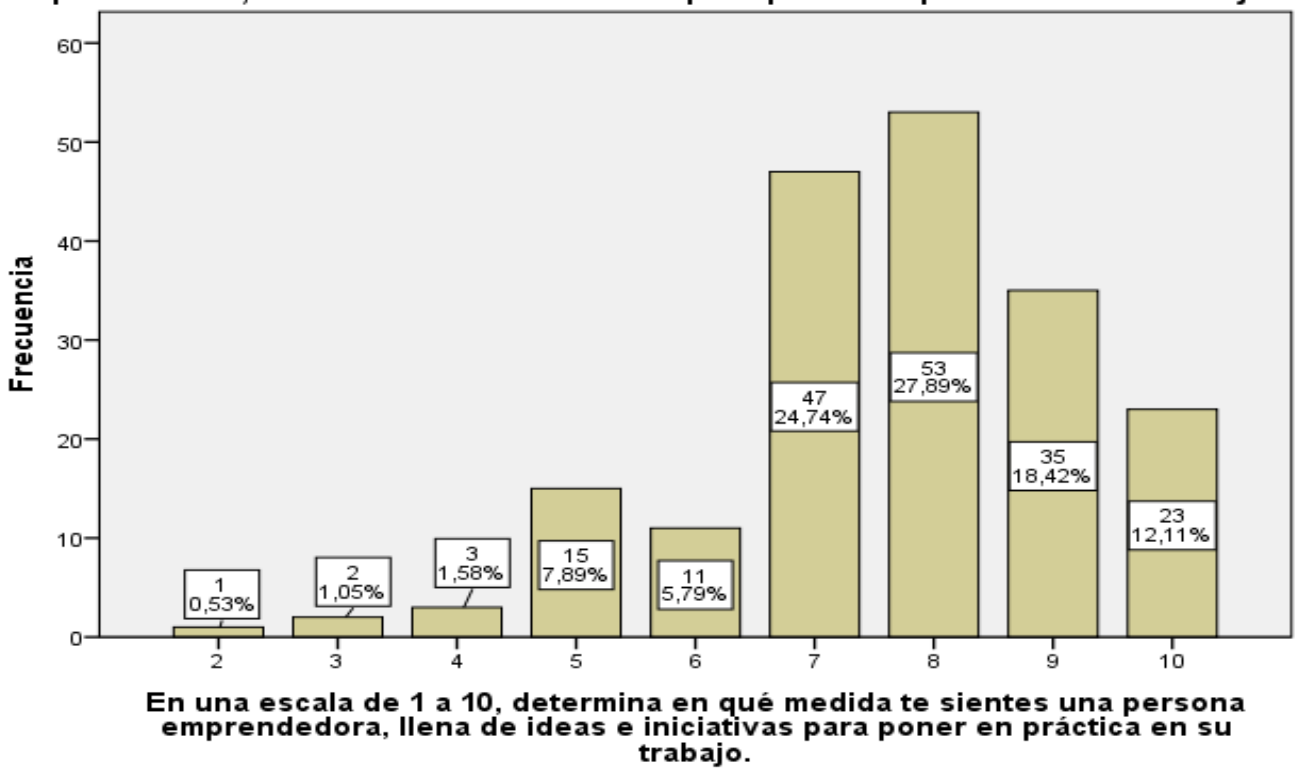

En contraparte, y aunque en la autoevaluación emprendedora la mayor parte del alumnado se consideran personas emprendedoras, y que según la Figura 2, casi en su totalidad $(n=188)$, desean fundar una empresa propia, $108(57,45 \%)$ de estos, piensan que, en contraste a décadas antecesoras, es más difícil en la actualidad crear una empresa; situación que probablemente acontece al complejo dinamismo del sector empresarial (Boja, et al., 2020), y a la falta de sistemas de incentivos de carácter de financiamiento (Zisis, et al., 2017) y últimamente la presencia del covid19 que redujo sustancialmente desde marzo de 2020 a septiembre de ese mismo año las ventas por el confinamiento obligatorio de los meses iniciales. No así, los 80 (42,55\%) estudiantes restantes, que desean crear su propia empresa, expresaron que es más fácil crear una empresa en comparación con las décadas anteriores. 
Perfil emprendedor de los estudiantes de la carrera de Economía de la Universidad Técnica de Machala en presencia del COVID-19

\section{Figura 2}

\section{Dificultad y deseabilidad de crear una empresa}

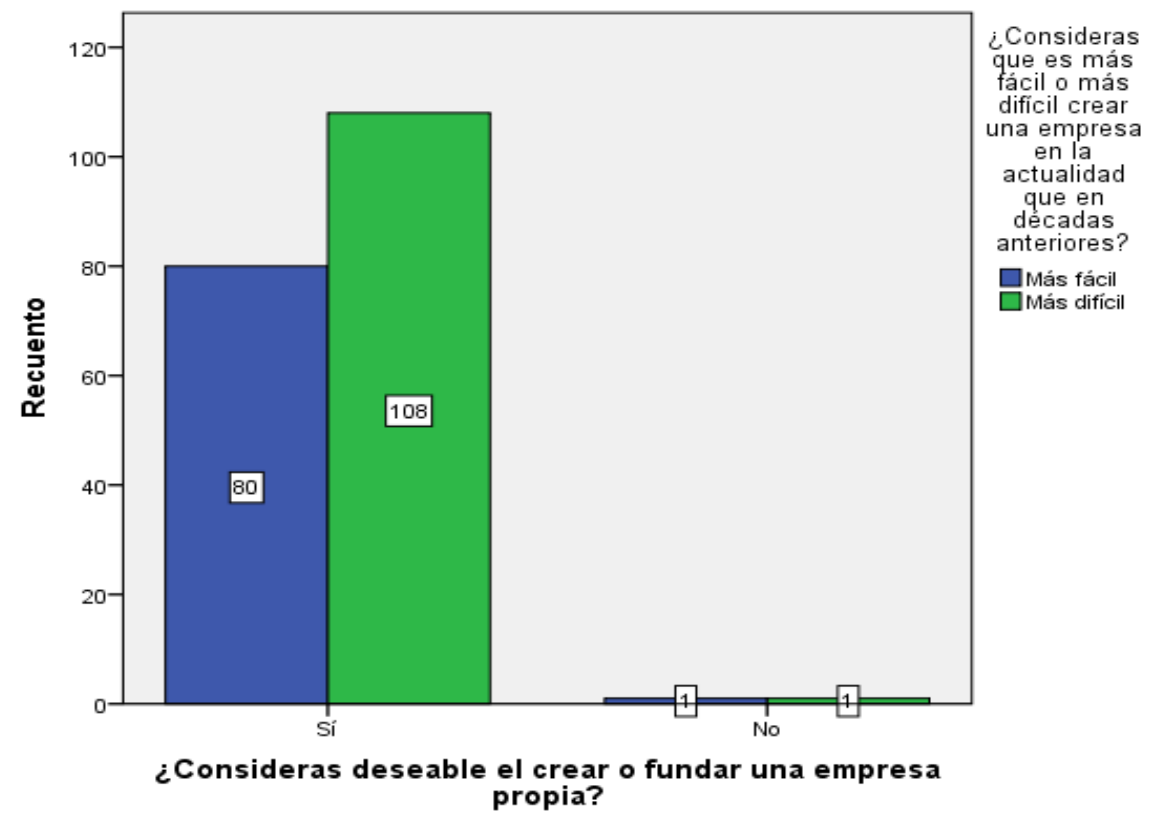

En adición, conforme a la percepción de los estudiantes, la mayor proporción señala, que las principales problemáticas con las que tienen que lidiar al momento de emprender y crear una empresa son (Figura 3): la falta de capital inicial $(n=116)$, y la situación económica $(n=112)$; seguido del temor al fracaso $(n=76)$, la falta de conocimiento y experiencia $(n=75)$, el elevado riesgo del fracaso empresarial $(n=74)$, y las cargas fiscales impuestas por el Estado $(n=71)$.

\section{Figura 3}

\section{Problemas para la creación de una empresa}

Falta de conocimiento y experiencia

$$
\begin{array}{r}
\text { Temor al fracaso } \\
\text { Cargas fiscales } \\
\text { Falta de capital inicial } \\
\text { Situación económica }
\end{array}
$$

Riesgo demasiado elevado
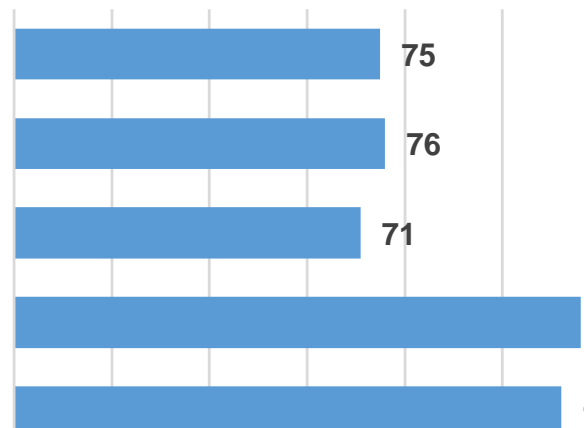

116
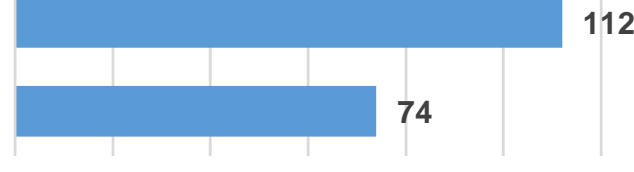

0

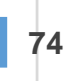

74

$\begin{array}{lllllll}20 & 40 & 60 & 80 & 100 & 120 & 140\end{array}$

Esta obra se comparte bajo la licencia Creative Common Atribución-No Comercial 4.0 International (CC BY-NC 4.0) Revista de la Universidad Internacional del Ecuador. URL: https://www.uide.edu.ec/ 
Complementando a lo anteriormente descrito, en la Figura 4, se muestra la autovaloración de 20 diferentes cualidades que conforman el perfil emprendedor, de acuerdo a una de las aristas del modelo de (Veciana y Urbano, 2004), en la cual, la mayor cantidad de los valores medios obtenidos, se acercan al máximo de 4 puntos (sí, siempre); siendo las características más destacables, en términos de mejor puntuación, la capacidad de los universitarios de economía para asumir la responsabilidad de sus decisiones, sean estas acertadas o erradas; seguido del gusto por sentirse independientes, la capacidad de autodisciplina, el entusiasmo, y la perseverancia.

Por otra parte, los atributos con menor puntuación fueron: la tolerancia al fracaso, el afrontamiento a las dificultades, y a la estabilidad emocional. Este comportamiento denota similitud con lo hallado en la investigación de Rodriguez y Fuentes (2013), aplicada a los estudiantes de la Universidad de Córdova.

\section{Figura 4}

Perfil emprendedor de los estudiantes de economía

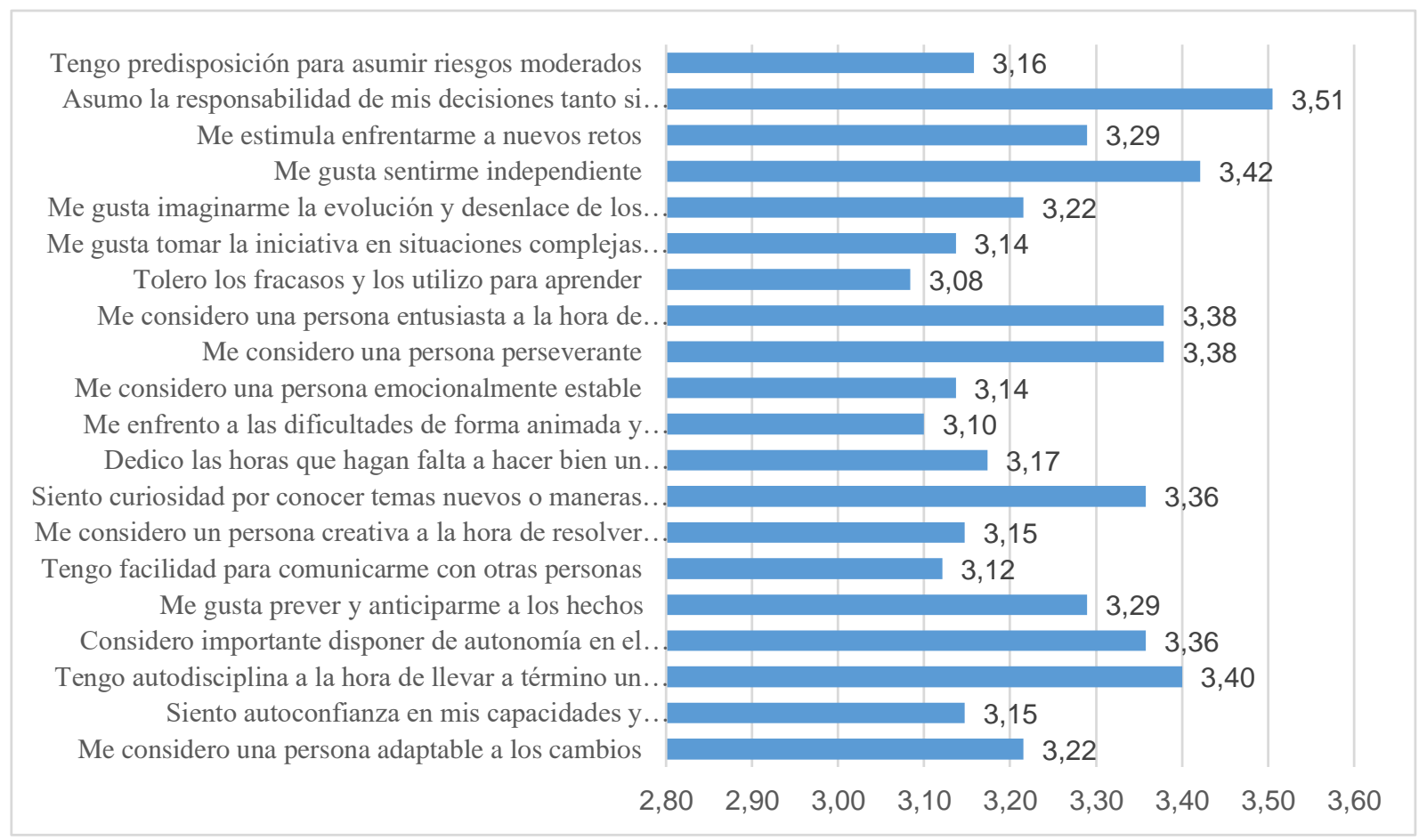

De modo complementario, en la Tabla 4, se avista la correlación existente entre el perfil emprendedor y la autovaloración emprendedora del alumnado de la carrera de economía, la que, según el test de correlación de Pearson, se encuentran asociadas con una linealidad positiva, a un nivel de significancia bilateral del 1\%, de forma moderada al 40,9\% (Roy, Rivas, Pérez, y Palacios, 2019). 
Perfil emprendedor de los estudiantes de la carrera de Economía de la Universidad Técnica de Machala en presencia del COVID-19

\section{Tabla 4}

\section{Correlación de Pearson}

\begin{tabular}{llr}
\hline & & Autovaloración emprendedora \\
\hline Perfil emprendedor & Correlación &, $409^{* *}$ \\
& Sig. (bilateral) & 0 \\
& N & 190 \\
\hline
\end{tabular}

**. La correlación es significativa en el nivel 0,01 (bilateral).

Finalmente, y en cumplimiento al objetivo planteado, mediante la prueba no paramétrica ${ }^{2}$ de análisis de varianza Kruskal Wallis, con un valor del estadístico de 87,5\%, se determinó que no existe diferencia estadísticamente significativa en el perfil emprendedor de los estudiantes de los diferentes semestres de la Carrera de Economía; debido presumiblemente, a que durante toda la Carrera (según la malla curricular), el alumnado cursa asignaturas y complementos formativos que fortalecen el componente del emprendimiento.

\section{Tabla 4}

Análisis de varianza no paramétrica

\begin{tabular}{lc}
\hline \multicolumn{2}{c}{ Kruskal Wallis } \\
\hline Sig. & 0,875 \\
Chi^2 $^{\wedge}$ & 3,103 \\
\hline Semestres & Media \\
\hline Cuarto & 2,94 \\
Segundo & 3,11 \\
Quinto & 3,19 \\
Tercero & 3,25 \\
Primero & 3,27 \\
Octavo & 3,30 \\
Séptimo & 3,31 \\
Sexto & 3,40 \\
\hline
\end{tabular}

\section{Conclusiones}

La muestra tomada de la Carrera de Economía de la Universidad Técnica de Machala, indica que hay un mayor peso de respuestas en los semestres superiores. El promedio de 7.6 sobre un máximo de 10, indica que los estudiantes se perciben como emprendedores, y tienen el deseo de tener su propia empresa; aunque, se muestran temerosos por problemas habituales que se palpan

\footnotetext{
2 No cumplió con dos (homocedasticidad e independencia) de las tres condiciones (normalidad, homocedasticidad, e independencia) para la validación de un modelo paramétrico (anova).
} 
al momento de emprender, como la falta de financiamiento, la situación económica (agravada por el COVID-19), el temor al fracaso, y los impuestos.

El perfil emprendedor encontrado en la muestra, define al emprendedor de la Carrera de Economía como responsable de sus decisiones, independiente, autodisciplinado, entusiasta, perseverante, curioso, autónomo, con capacidad de enfrentarse a retos, y adaptable a los cambios, pese a que no se muestran muy tolerables al fracaso, y al afrontamiento de las dificultades.

Algo importante de destacar, es que, pese a que el perfil emprendedor de los estudiantes se muestra óptimo, este correlaciona moderadamente con su autovaloración emprendedora; así como también la no existencia de diferencia estadísticamente significativa en el perfil emprendedor entre los estudiantes de todos semestres analizados; lo que lleva a reflexionar si los contenidos estudiados por los alumnos tienen los contenidos suficientes para que durante o a posteriori de su formación profesional puedan entender mejor las oportunidades de emprender y de superar obstáculos iniciales en la decisión de crear una empresa; dejando abierta una arista para futuras investigaciones que reporten si los contenidos entregados por la Carrera de Economía sirven o no a los estudiantes que buscan emprender; así como la producción de emprendimientos por sector de oportunidades, el porcentaje de uso y el aprovechamiento de las asignaturas en la propuesta final de valor.

\section{Referencias bibliográficas}

Bagheri, A., \& Lope Pihie, Z. A. (2013). Role of University Entrepreneurship Programs in Developing Students' Entrepreneurial Leadership Competencies: Perspectives From Malaysian Undergraduate Students. Journal of Education for Business, 88(1), 51-61. https://doi.org/10.1080/08832323.2011.638681

Bernal, A., y Cárdenas, A. R. (2017). Evaluación del potencial emprendedor en escolares. Una investigación longitudinal. Educación $\quad X X 1, \quad 20(2), \quad 73-94$. https://doi.org/10.5944/educXX1.14162

Boja, A., Carvajal, H., y Vite, H. (2020). Modelo de emprendimiento y análisis de los factores determinantes para su sostenibilidad. Revista ESPACIOS. 41(24). 183-196. http://www.revistaespacios.com/a20v41n24/20412415.html

Bagheri, A., \& Lope Pihie, Z. A. (2013). Role of University Entrepreneurship Programs in Developing Students' Entrepreneurial Leadership Competencies: Perspectives from Malaysian Undergraduate Students. Journal of Education for Business, 88(1), 51-61. https://doi.org/10.1080/08832323.2011.638681

Boza, J., Mendoza, E., y Intriago, E. (2020). La educación en emprendimiento de los estudiantes de las carreras empresariales de la Universidad Técnica Estatal de Quevedo. Conrado, 16(72), 7-14. $\quad$ http://scielo.sld.cu/scielo.php?script=sci_arttext\&pid=S1990$\underline{86442020000100007}$

Bravo, I., Bravo, M., Preciado, J., y Mendoza, M. (2021). Educación para el emprendimiento y la intención de emprender. Revista Economía y Política (33), 1-17. https://publicaciones.ucuenca.edu.ec/ojs/index.php/REP/article/view/3492/2548

CEAACES. (2018). Ley Orgánica de Educación Superior, LOES. In 2012. https://bit.ly/3frnbS9 
Perfil emprendedor de los estudiantes de la carrera de Economía de la Universidad Técnica de Machala en presencia del COVID-19

Charrón Vías, M., \& Rivera-Cruz, B. (2020). Fostering innovation and entrepreneurial culture at the business school: A competency-based education framework. Industry and Higher Education, 34(3), 160-176. https://doi.org/10.1177/0950422219895209

Chaves, E., y Rodríguez, L. (2018). Análisis de confiabilidad y validez de un cuestionario sobre entornos personales de aprendizaje (PLE). Ensayos Pedagógicos, 13(1), 71-106. https://dialnet.unirioja.es/servlet/articulo?codigo $=7038088$

Febriyantoro, M. T. (2019). the Role of Entrepreneurial Campus in Establishing of Students' Entrepreneurial Mindset and Entrepreneurial Spirit. 141-146. https://doi.org/10.31219/osf.io/r35nh

Flores, E., Miranda, M., y Villasís, M. (2017). El protocolo de investigación VI: cómo elegir la prueba estadística adecuada. Estadística inferencial. Revista alergia México, 64(3), 364370. https://doi.org/10.29262/ram.v64i3.304

Formichella, M. M. (2004). El concepto de emprendimiento y su relación con la educación, el empleo y el desarrollo local. In Serie Instituto Nacional de Tecnología Agropecuaria (INTA).

García, J., Reding, A., y López, J. (2013). Cálculo del tamaño de la muestra en investigación en educación médica. Investigación en educación médica, 2(8), 217-224. http://www.scielo.org.mx/pdf/iem/v2n8/v2n8a7.pdf

González, G., Becerril, M., y Fonseca, A. (2018). El engagement como factor de formación y desarrollo de la cultura emprendedora en estudiantes universitarios. IE Revista de investigación educativa de la REDIECH, 9(17), 103-118. http://www.scielo.org.mx/scielo.php?script=sci_arttext\&pid=S2448$\underline{85502018000200103}$

González, M., Rodríguez, L., y Pérez, M. (2017). Efecto de los programas de educación en emprendimiento sobre la intención emprendedora de los estudiantes universitarios. Economía Industrial 93-103. https://dialnet.unirioja.es/servlet/articulo?codigo $=6117396$

Haase, H., \& Lautenschläger, A. (2011). The Myth of Entrepreneurship Education: Seven arguments against teaching business. Journal of Entrepreneurship Education, 14, 147-162.

Harrison, J., \& Turok, I. (2017). Universities, knowledge and regional development. Regional Studies, 51(7), 977-981. https://doi.org/10.1080/00343404.2017.1328189

Hernández, J., Espinosa, F., Rodríguez, J., y Chacón, J. (2018). Sobre el uso adecuado del coeficiente de correlación de Pearson: definición, propiedades y suposiciones. Archivos Venezolanos de Farmacología y Terapéutica, 37(5), 587-601. https://www.redalyc.org/jatsRepo/559/55963207025/55963207025.pdf

Hernández, R., Fernández, C., y Baptista, P. (2018). Metodología de la investigación. México D.F.: McGraw Hill.

Herrera, M., y Konic, P. (2017). Conocimiento del profesor sobre la importancia del muestreo aleatorio simple para la estimación de parámetros. Actas del Segundo Congreso International Virtual sobre el Enfoque Ontosemiótico del Conocimiento y la Instrucción Matemáticos, 1-10. http://enfoqueontosemiotico.ugr.es/civeos/herrera_konic.pdf

Honig, B. (2004). Entrepreneurship Education: Toward a Model of Contingency-Based Business Planning. Academy of Management Learning and Education, 3(3), 258-273. https://doi.org/10.5465/amle.2004.14242112 
Lasio, V., Amaya, A., Zambrano, J., \& Ordeñana, X. (2020). Global Entrepreneurship Monitor (Issue 13903047). content/uploads/2021/02/GEM_Ecuador_2019.pdf

https://www.espae.edu.ec/wp-

León, A., y Pérez, C. (2019). Análisis estadístico en investigaciones positivas: medidas de tendencia central. Revista Científica Electrónica de Ciencias Humanas, 15(43), 50-60. http://bdigital2.ula.ve:8080/xmlui/handle/654321/3770

Leutner, F., Ahmetoglu, G., Akhtar, R., \& Chamorro-Premuzic, T. (2014). The relationship between the entrepreneurial personality and the Big Five personality traits. Personality and Individual Differences, 63, 58-63. https://doi.org/10.1016/j.paid.2014.01.042

López Núñez, M. I., Rubio Valdehita, S., Díaz Ramiro, E. M., y Martín Seoane, G. (2021). Perfil emprendedor de estudiantes universitarios: un modelo predictivo. Revista de Educación. https://doi.org/10.4438/1988-592X-RE-2021-392-477

Malinda, M. (2019). Learning methods of business plan subject to increase entrepreneurial skill, entrepreneurial intention and entrepreneurial spirit of students. International Journal of Information and Education Technology, 9(11), 810-814. https://doi.org/10.18178/ijiet.2019.9.11.1309

Martin, B. C., McNally, J. J., \& Kay, M. J. (2013). Examining the formation of human capital in entrepreneurship: A meta-analysis of entrepreneurship education outcomes. Journal of Business Venturing, 28(2), 211-224. https://doi.org/10.1016/j.jbusvent.2012.03.002

Moran-Montalvo, C., y Sánchez-Riofrío, A. (2018). El perfil emprendedor universitario: El caso de la Universidad de Especialidades Espíritu Santo en Ecuador. Revista Ciencia Administrativa, 1. https://www.uv.mx/iiesca/files/2018/11/10CA201801.pdf

Niño, V. (2019). Metodología de la investigación: diseño, ejecución e informe. Bogotá: Ediciones de la U.

Núñez, C. (2018). Análisis de varianza no paramétrica: un punto de vista a favor para utilizarla. Acta agrícola y pecuaria, 4(3), 69-79. https://doi.org/10.30973/aap/2018.4.3/1

Otzen, T., y Manterola, C. (2017). Técnicas de Muestreo sobre una Población a Estudio. International Journal of Morphology, 35(1), 227-232. http://doi.org/10.4067/S071795022017000100037

Rodríguez, M., y Mendivelso, F. (2018). Diseño de investigación de corte transversal. Rev. Médica. Sanitas (21), 141-146.

Rodriguez, P., y Fuentes, F. (2013). Estudio sobre el Perfil Emprendedor en el Alumnado Universitario de Córdoba (III edición). Córdoba: Consejo social de la Universidad de Córdoba. https://bit.ly/3E65cdL

Roy, I., Rivas, R., Pérez, M., y Palacios, L. (2019). Correlación: no toda correlación implica causalidad. Revista alergia México, 66(3), 354-360. https://doi.org/10.29262/ram.v66i3.651

Sánchez, F. (2019). Fundamentos epistémicos de la investigación cualitativa y cuantitativa: Consensos y disensos. Revista Digital de Investigación en Docencia Universitaria, 13(1), 102-122. https://bit.ly/3rbVKlc

Simancas, M., y Arévalo, L. (2017). Desempeño de cuatro métodos estadísticos para evaluación de la concordancia prueba-reprueba de variables continuas en una muestra. Revista Biosalud, 16(1), 19-29. https://doi.org/10.17151/biosa.2017.16.1.4

Valdivieso, C., Valdivieso, R., y Valdivieso, O. (2011). Determinación del tamaño muestral mediante el uso de árboles de decisión. UPB - Investigación \& desarrollo, 148-176. ftp://ftp.repec.org/opt/ReDIF/RePEc/iad/wpaper/0311.pdf 
Perfil emprendedor de los estudiantes de la carrera de Economía de la Universidad Técnica de Machala en presencia del COVID-19

Veciana, J. M., Aponte, M., \& Urbano, D. (2005). University Students' Attitudes Towards Entrepreneurship: A Two Countries Comparison. The International Entrepreneurship and Management Journal, 1(2), 165-182. https://doi.org/10.1007/s11365-005-1127-5

Veciana, J., y Urbano, D. (2004). Actitudes de los estudiantes universitarios hacia la creación de empresas: un estudio. El emprendedor innovador y la creación de empresas, 35-58.

Vera Salazar, P., Amaru Galvis, E., y González Zabala, M. (2013). Concretando la tercera misión $(3 \mathrm{~m})$ de la Universidad Pública Regional. Impactos y percepciones de un proyecto de extensión. Caso Universidad del Magdalena. Clío América, 7(14), 135-152.

Víctor, H., José, M., y Nora, I. (2019). Universidad para el emprendimiento. Perfil formativo profesional y vocación de crear empresas. Universidad Técnica de Ambato, Ecuador. Revista Venezolana de Gerencia, 24(85), 31-47. https://doi.org/10.31876/revista.v24i85.23827

Zisis, N., Moya, P., y Molina, F. (2017). Percepciones de académicos sobre las dificultades para el fomento de la innovación y el emprendimiento: el caso de la FCFM de la Universidad de Chile. Journal of technology management \& innovation, 12(4), 97-105. https://scielo.conicyt.cl/scielo.php?pid=S071827242017000400010\&script=sci_arttext\&t $\underline{\operatorname{lng}=\mathrm{en}}$ 


\section{Anexos}

\section{Preguntas utilizadas del cuestionario actitudes hacia la creación de empresas de (Veciana y Urbano, 2004)}

$>$ ¿Consideras deseable el crear o fundar una empresa propia?

$>$ ¿Consideras que es más fácil o más difícil crear una empresa en la actualidad que en décadas anteriores?

$>$ En una escala de 1 a 10, determina en qué medida te sientes una persona emprendedora, llena de ideas e iniciativas para poner en práctica en su trabajo.

Ítems de escala tipo Likert: $1=$ no, nunca; $2=$ bastante, a menudo; $3=$ poco, alguna vez; y 4= sí, siempre

$>$ ¿Me considero una persona adaptable a los cambios?

$>$ ¿Siento autoconfianza en mis capacidades y posibilidades personales y profesionales?

$>$ ¿Tengo autodisciplina a la hora de llevar a término un trabajo?

$>\quad$ ¿Considero importante disponer de autonomía en el trabajo?

$>$ ¿Me gusta prever y anticiparme a los hechos?

$>$ ¿Tengo facilidad para comunicarme con otras personas?

$>$ ¿Me considero una persona creativa a la hora de resolver los problemas?

$>$ ¿Siento curiosidad por conocer temas nuevos o maneras diferentes de hacer las cosas?

$>$ ¿Dedico las horas que hagan falta a hacer bien un trabajo o tirar adelante un proyecto?

$>\quad$ ¿Me enfrento a las dificultades de forma animada y optimista?

$>$ ¿Me considero una persona emocionalmente estable?

$>$ ¿Me considero una persona perseverante?

$>$ ¿Me considero una persona entusiasta a la hora de iniciar nuevos proyectos?

$>$ ¿Tolero bien los fracasos y los utilizo para aprender?

$>$ ¿Me gusta tomar la iniciativa en situaciones complejas o delicadas?

$>\quad$ ¿Me gusta imaginarme la evolución y desenlace de los proyectos que empiezo?

$>$ ¿Me gusta sentirme independiente?

$>$ ¿Me estimula enfrentarme a nuevos retos?

$>$ ¿Asumo la responsabilidad de mis decisiones tanto si son acertadas como erróneas?

$>$ ¿Tengo predisposición para asumir riesgos moderados? 